\title{
Can shear-wave elastography predict response to neoadjuvant chemotherapy in women with invasive breast cancer?
}

\author{
A Evans ${ }^{*}, 1$, S Armstrong ${ }^{2}$, P Whelehan ${ }^{1}, \mathrm{~K}$ Thomson $^{3}, \mathrm{P}$ Rauchhaus ${ }^{4}, \mathrm{C}$ Purdie ${ }^{5}, \mathrm{~L}$ Jordan $^{5}, \mathrm{~L} \mathrm{Jones}^{6}$, \\ A Thompson ${ }^{1}$ and S Vinnicombe ${ }^{1}$ \\ ${ }^{1}$ Dundee Cancer Centre, Ninewells Hospital and Medical School, Dundee DD1 9SY, UK; ${ }^{2}$ Department of Oncology, Ninewells \\ Hospital, Dundee DD1 9SY, UK; ${ }^{3}$ Department of Breast Imaging, Ninewells Hospital, Dundee DD1 9SY, UK; ${ }^{4}$ The Division of \\ Clinical and Population Sciences and Education, Ninewells Hospital, Dundee DD1 9SY, UK; ${ }^{5}$ Department of Pathology, Ninewells \\ Hospital, Dundee DD1 9SY, UK and 'Tumour Biology Laboratory' Barts Cancer Institute, Queen Mary University of London, \\ London EC1M 6BQ, UK
}

Background: Response of invasive breast cancer to neoadjuvant chemotherapy (NAC) is variable, and prediction of response is imperfect. We aimed to ascertain whether tissue stiffness in breast cancers, as assessed by shear-wave elastography (SWE) before treatment, is associated with response.

Methods: We retrospectively compared pre-treatment tumour mean tissue stiffness, with post-treatment Residual Cancer Burden (RCB) scores and its components in 40 women with breast cancer treated by NAC using Pearson's correlation coefficient (CC), a general linear model and multiple linear regression. Subgroup analysis was carried out for luminal, HER2-positive and basal immuno-histochemical subtypes.

Results: Statistically significant correlations were shown between stiffness and RCB scores and between stiffness and percentage tumour cellularity. The correlation between stiffness and percentage cellularity was strongest $(C C 0.35(P<0.0001)$ compared with CC $0.23(P=0.004)$ for the RCB score). The results of a general linear model show that cellularity and RCB score maintain independent relationships with stiffness. By multiple linear regression, only cellularity maintained a significant relationship with stiffness.

Conclusion: Pre-treatment tumour stiffness measured by SWE, has a statistically significant relationship with pathological response of invasive breast cancer to NAC.

Response to neoadjuvant therapy is variable and prediction of response is currently poor. Validated biomarkers predictive of response/resistance prior to the commencement of neoadjuvant chemotherapy (NAC) are not currently routinely used, in contrast to endocrine neoadjuvant therapy. Predictive biomarkers for chemotherapy would allow the women most likely to benefit from NAC to do so, while sparing women unlikely to respond from considerable morbidity. Research in this area has so far been inconclusive but genomic predictors, and the immuno-histochemical/molecular characteristics of the tumour have been considered (Desmedt et al, 2011; Hatzis et al, 2011; Lips et al, 2012). Recent work has shown that stromal gene signatures are powerful predictors of response to NAC (Farmer et al, 2009) and that the responses of cancer-associated fibroblasts to chemotherapy are very variable (Tokes et al, 2009). This suggests that both tumoural and stromal factors may be important in predicting resistance to NAC. Two imaging modalities - magnetic resonance imaging (MRI) and elastography - commonly show abnormalities in the stroma surrounding breast cancers 
(Hattangadi et al, 2008; Athanasiou et al, 2010; Evans et al, 2010; Chang et al, 2011; Berg et al, 2012). Magnetic resonance imaging shows subtle contrast enhancement of surrounding stroma in some cases and this enhancement in one small series has been shown to be predictive of response to NAC (Hattangadi et al, 2008). A feature of malignancy on ultrasound elastography is stiffness extending from the greyscale abnormality into the surrounding stroma. Breast cancers stiff on strain elastography were less likely to have a pathological complete response after NAC than cancers which were soft on elastography (Hayashi et al, 2012). Shear-wave elastography (SWE), unlike strain elastography, is quantitative and highly reproducible (Evans et al, 2010; Cosgrove et al, 2012).

Residual Cancer Burden (RCB) scoring is a method of assessing pathological response to NAC, as a continuous variable, and it is derived from the post-chemotherapy primary tumour dimensions, cellularity of the tumour bed and axillary lymph node burden. The RCB score yields additional prognostic information compared with the presence or absence of a pathological complete response (Symanns et al, 2007). We therefore aimed to ascertain whether the degree of tissue stiffness in breast cancers, as assessed by SWE before treatment, was associated with the response to NAC, as assessed by the RCB score.

\section{MATERIALS AND METHODS}

Eligible patients, identified from clinical records, were those who had been treated with NAC and subsequent surgical excision for primary invasive breast cancer between 10 May 2010 and 23 Febuary 2013. All 40 patients identified had undergone ultrasound SWE using an Aixplorer ultrasound system (SuperSonic Imagine, Aix en Provence, France), as standard of care in our clinic since April 2010 for women presenting with symptomatic or screen-detected abnormalities. The scans were performed by one of the four practitioners with between 5 and 20 years of breast ultrasound experience and at least 3 months' previous experience of performing SWE on solid breast lesions.

In accordance with UK National Research Ethics Service guidance (National Research Ethics Service, 2008), ethical approval for the study was waived as the study was carried out retrospectively on routinely acquired data available to the principal investigator by virtue of his clinical role. However, written informed consent to use images was obtained, according to routine procedure in our institution. Combined greyscale and elastography examination times were between 10 and $20 \mathrm{~min}$. Acquisition of the elastography images takes 1-2 min. The mean stiffness measurements were generated by reviewing the images after the examination. This data extraction took 1-2 min per lesion.

Four elastography images taken in two orthogonal planes were obtained for each lesion by holding the transducer still, with no pressure being applied, and allowing the image to build up over about $10 \mathrm{~s}$. The mean stiffness measurements were obtained by moving a delineated region of interest (ROI) over the colour map. As the ROI moves, the figures change in real time so the ROI can be positioned over the stiffest tissue shown on the image (Figure 1). Because many cancers are not uniformly stiff but have a halo of peri-tumoural stromal stiffness, the ROI was kept as small as possible $(2 \mathrm{~mm})$ to allow measurement of the stiffest tissue anywhere within the lesion or in the peri-tumoural stroma. One stiffness value per image was measured. The values used for the analysis were the mean elasticity measurements in kilopascals $(\mathrm{kPa})$ within the ROI, once placed over the stiffest area. The individual values from each of the four images were used for analysis (they were not averaged).

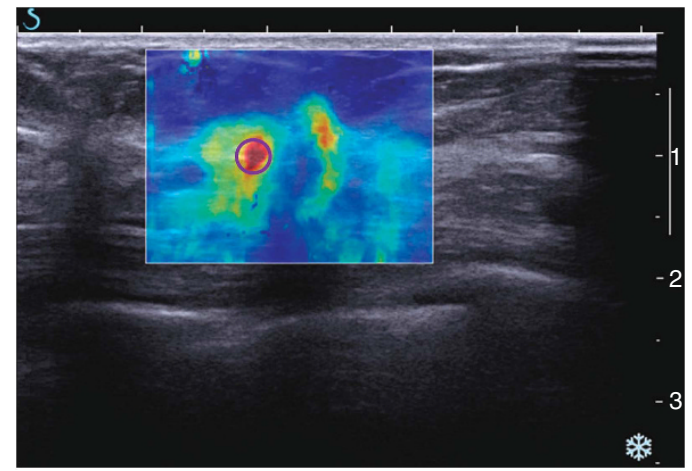

Figure 1. Shear-wave elastography image showing a $2 \mathrm{~mm}$ ROI placed over the stiffest region on this image of a cancer from the study. The mean stiffness value was $125 \mathrm{kPa}$ and the RCB score after chemotherapy was 0 .

Of the 40 patients in the study, 25 were treated with six cycles of FEC (5FU, epirubicin and cyclophosphamide); one patient's disease progressed after three cycles and she proceeded directly to surgery. Two patients received FEC and Docetaxel, 12 patients received trastuzumab with Docetaxel, either alone (3 patients) or after three cycles of FEC ( 9 patients) and 1 patient received five cycles of Docetaxel, TDM1 and pertuzumab.

The mean elasticity measurements from the SWE scans performed prior to NAC were compared with the RCB scores and RCB score components, assessed from the resection specimens by a consultant specialist breast pathologist. The components assessed were the percentage invasive cancer cellularity, number of positive lymph nodes and the size of the largest axillary metastasis. The pathologist was blinded to the SWE findings. Tumours were divided into luminal, HER-2 positive and basal subgroups on the basis of immuno-histochemistry in order to detect whether any associations found were limited to particular tumour subgroups. The RCB scores and components thereof were correlated with pretreatment mean stiffness readings using Pearson's correlation coefficient (CC). The independence of relationships between the RCB scores and its components with stiffness was assessed using a general linear model and multiple linear regression. We also compared the RCB scores and components with age, as a potential confounding variable. For the statistical analysis, all the elasticity results from all images were used. Tests for normality of the outcome variable were performed prior to analysis. Correlations between all the co-variables were calculated, and univariate associations between stiffness and co-variables was established using linear regression. A final mixed model investigated the influence of the co-variables on stiffness, taking into account the repeated measures.

A value of $P<0.05$ was deemed to indicate a statistically significant finding.

\section{RESULTS}

All 40 women had presented with symptoms and were aged between 36.6 and 68.4 years (mean 50.2 years). The pre-treatment mean tumour size as assessed by greyscale ultrasound was $28 \mathrm{~mm}$ (range $11-50 \mathrm{~mm}$ ). Correlations between mean pre-treatment stiffness readings and RCB scores and its components and age are shown in Table 1. Statistically significant associations are shown between mean stiffness and RCB scores and between mean stiffness and percentage tumour cellularity. The correlation between mean stiffness and percentage cellularity is strongest (CC $0.35(P<0.0001)$ compared with CC $0.23(P=0.004)$ for the 
Table 1. Correlations between pre-treatment stiffness readings and age, RCB score and RCB score components in 40 patients

\begin{tabular}{|l|l|c|c|}
\hline Reference & Comparison & $\begin{array}{c}\text { Correlation } \\
\text { coefficient }\end{array}$ & $\begin{array}{c}\text { P-value } \\
\text { correlation }\end{array}$ \\
\hline Stiffness reading & Age & 0.19 & 0.018 \\
\hline & Cellularity (\%) & 0.35 & $<0.0001$ \\
\hline & Number of positive nodes & 0.047 & 0.56 \\
\hline & RCB score & 0.23 & 0.0044 \\
\hline & Size of largest axillary metastasis & 0.14 & 0.07 \\
\hline & Invasive size & 0.12 & 0.14 \\
\hline & \multicolumn{3}{|l}{} \\
\hline
\end{tabular}

RCB score). These relationships are shown graphically in Figures 2 and 3. Statistically significant correlations were not shown between mean stiffness and other components of the RCB score, that is, the number of lymph nodes involved, the size of the largest axillary metastasis.

The results of the general linear model are shown in Table 2. Cellularity and RCB score maintain independent relationships with mean stiffness. Following multiple linear regression of the relationships between mean stiffness and RCB score and mean stiffness and cellularity, only cellularity maintained a statistically significant relationship with mean stiffness (Table 3 ).

Correlations between stiffness and RCB scores and cellularity for each breast cancer subgroup are shown in Table 4 . $R$ values for stiffness $v s$ RCB scores varied from 0.13 to 0.42 and for stiffness $v s$ cellularity from 0.30 to 0.65 . The correlation between cellularity and stiffness for the HER-2 positive was statistically significant. The distributions of RCB score and residual cellularity by histological grade and histological type are shown in Table 5.

\section{DISCUSSION}

We have found that pre-treatment tissue elasticity in breast cancer, measured by ultrasound SWE, has a statistically significant relationship with the subsequent response of invasive breast cancer to NAC. This relationship was seen across the common luminal and HER 2 + subgroups of breast cancer, as defined by immunohistochemistry, but numbers in each subgroup were small. Our findings are concordant with a previous study which found that tumour stiffness assessed by scoring strain elastography images was related to the proportion of women receiving NAC in whom a pathological complete response was achieved (Hayashi et al, 2012). This previous study had the advantage of a larger study group, whereas the current study has the advantage of using a more reproducible technique which gives a result as a continuous numerical variable rather than a dichotomised yes/no result. Our study has also used a validated method of assessing response to chemotherapy which has been shown on multivariate analysis to yield richer prognostic information than that given by the proportion women of patients having a pathological complete response. The correlation we have shown between tumour stiffness prior to treatment and reduction in tumour cellularlity after treatment although statistically significant is weak. This suggests that it will be of little clinical use in its current form. It is hoped that in the future tumour stiffness could be part of a multimodal model which might yield more clinically useful predictions. It should also be stressed that tumour stiffness only predicts reduction in cellularity of the primary tumour and has no value in predicting the response of nodal metastases to NAC.

The association between stromal stiffness and response to NAC makes sense biologically as stromal gene signatures are predictive

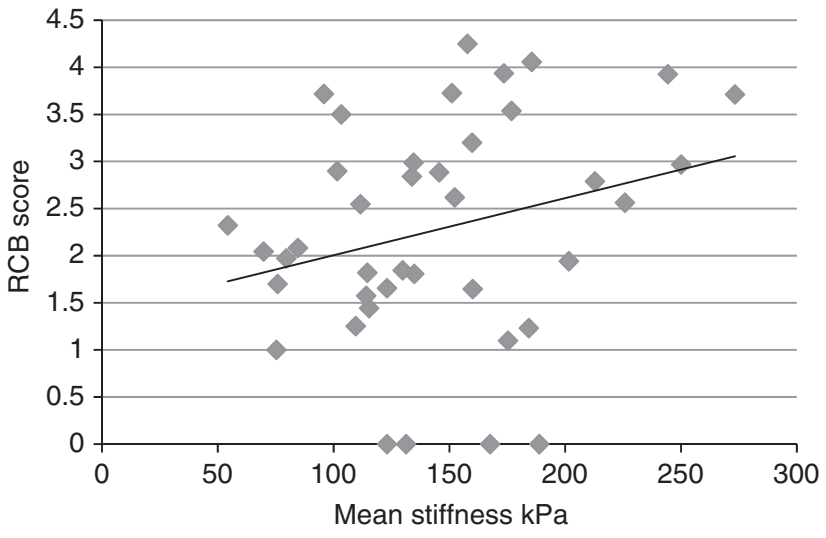

Figure 2. Relationship between pre-treatment mean stiffness and post-treatment RCB score.

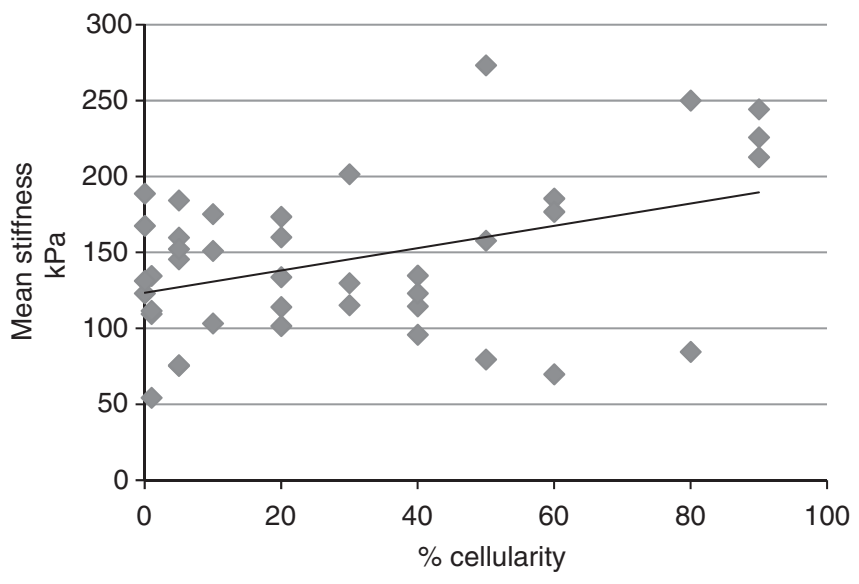

Figure 3. Relationship between pre-treatment mean stiffness and percentage residual cellularity after treatment.

Table 2. Results of a general linear model assessing independence of relationships of age, RCB scores and RCB score components to stiffness in 40 patients

\begin{tabular}{|l|c|c|}
\hline Reference & F value & $\boldsymbol{P}$-value \\
\hline Age & 1.95 & 0.17 \\
\hline Cellularity (\%) & 7.58 & 0.01 \\
\hline Molecular type & 0.39 & 0.68 \\
\hline Number of positive nodes & 0.35 & 0.56 \\
\hline RCB class & 2.46 & 0.08 \\
\hline RCB score & 4.36 & 0.04 \\
\hline Size of largest metastasis & 1.86 & 0.18 \\
\hline
\end{tabular}

The ANOVA F-test statistic denotes the ration of the between-group variance and the within-group variance, and as such a measure of the difference in data spread between the group measurements.

Table 3. Results of multiple linear regression analysis of the relationship of stiffness to cellularity and RCB score

\begin{tabular}{|l|c|c|}
\hline Reference & F value & $\boldsymbol{P}$-value \\
\hline Cellularity (\%) & 4.27 & 0.04 \\
\hline RCB score & 0.81 & 0.4 \\
\hline
\end{tabular}


Table 4. Correlation between stiffness and RCB scores and cellularity in breast cancer subgroups

\begin{tabular}{|l|c|c|c|c|}
\hline Reference & Cell type & Comparison & $\begin{array}{c}\text { Correlation } \\
\text { value }\end{array}$ & P-value \\
\hline Average stiffness & Basal & Cellularity (\%) & 0.30 & 0.22 \\
\hline & $N=18$ & RCB score & 0.32 & 0.18 \\
\hline & Luminal & Cellularity (\%) & 0.54 & 0.13 \\
\hline & $N=9$ & RCB score & 0.42 & 0.26 \\
\hline & HER-2 + & Cellularity (\%) & 0.65 & 0.01 \\
\hline & $N=13$ & RCB score & 0.13 & 0.67 \\
\hline
\end{tabular}

Table 5. Distribution of RCB score and residual cellularity by histological grade and histological type

\begin{tabular}{|l|c|c|c|}
\hline & Number & RCB score (mean) & $\begin{array}{c}\text { Residual cellularity } \\
\text { (mean) }\end{array}$ \\
\hline Grade 2 & 10 & 2.13 & 17.6 \\
\hline Grade 3 & 30 & 2.33 & 33.3 \\
\hline Ductal & 35 & 2.27 & 28.4 \\
\hline Lobular & 3 & 2.66 & 37 \\
\hline Other & 2 & 1.81 & 35 \\
\hline
\end{tabular}

of response to NAC. Therefore, stromal stiffness may be an imaging biomarker for the stromal structural abnormalities arising from such tumour-associated fibroblast stromal gene signatures (Farmer et al, 2009).

Other imaging techniques, such as MRI, have also been shown to yield information which relates to the sensitivity of breast cancer to NAC. Magnetic resonance imaging indices which have been suggested as being helpful in this regard include morphology, tumour enhancement characteristics, apparent diffusion coefficient, spectroscopy and peri-tumoural stromal enhancement (Hattangani et al, 2008; Cao et al, 2012; Dongfeng et al, 2012).

The extracellular matrix (ECM), of which collagen is a major component, has a critical role in the development and invasiveness of primary breast cancers. The stiffness in the peri-tumoural stroma appears to be due to the increased collagen cross-linking seen in cancer-associated stroma which leads to increased focal adhesion, enhanced PI3 kinase activity and induction of tumour invasion (Levental et al, 2009). Abnormal collagen has been shown to extend a number centimetres beyond breast cancers and this is concordant with the cancer-associated stiffness on SWE extending well beyond the tumour itself (Lewis et al, 2000). This in turn is related to a number of stromal biomarkers such a lysyl oxidase (LOX), fibronectin and caveolin 1. Lysyl oxidase is an ECM remodelling enzyme and appears to have roles in promoting cancer cell motility and invasion (Chen et al, 2012), whereas loss of stromal caveolin-1 in breast cancers is associated with early tumour recurrence, metastasis and drug resistance, leading to poor clinical outcome (Witkiewicz et al, 2009). This prognostic influence has been shown to be independent of the classic tumoural prognostic features.

The weaknesses of this first study of SWE in the NAC setting were the small number of patients and the heterogeneity among tumour types and treatment given. The study employed 2D SWE and thus has purely assessed the mean stiffness of the stiffest part of the tumour/peri-tumoural area. Three-dimensional SWE has recently been developed and this allows the volume of stiff tissue to be measured. The significance of the volume of abnormal stiffness associated with breast cancers has yet to be ascertained.

It would also be of great interest to compare peri-tumoural stiffness with stromal biomarkers such as collagen cross-linking, caveolin 1 and LOX. If correlations exist then the possibility would be raised of peri-tumoural stiffness being clinically valuable noninvasive, reproducible, predictive biomarker for poor clinical outcome, independent of the classical prognostic features of histological grade, nodal stage and invasive size.

Our anecdotal experience is that cancer-associated stiffness decreases during neoadjuvant therapy but this phenomenon has yet to be formally evaluated. It is possible that SWE may be useful in the assessment of response to neoadjuvant therapy as well being useful in predicting response.

In conclusion, we have found that pre-treatment tissue elasticity as measured by SWE has a significant relationship with the subsequent reduction in cellularity of the primary tumour in response to NAC.

\section{REFERENCES}

Athanasiou A, Tardivon A, Tanter M, Sigal-Zafrani B, Bercoff J, Deffieux T, Gennisson J, Fink M, Neuenschwander S (2010) Breast lesions: quantitative elastography with supersonic shear imaging - preliminary results. Radiology 256: 297-303.

Berg WA, Cosgrove DO, Dore CJ, Schäfer F, Svensson W, Hooley R, Ohlinger R, Mendelson E, Balu-Maestro C, Locatelli M, Tourasse C, Cavanaugh B, Juhan V, Stavros T, Tardivon A, Gay J, Henry J, Cohen-Bacrie C (2012) Shear-wave elastography improves the specificity of breast US: the BE1 multinational study of 939 masses. Radiology 262: 435-449.

Cao MD, Sitter B, Bathen TF, Bofin A, Lønning PE, Lundgren S, Gribbestad IS (2012) Predicting long-term survival and treatment response in breast cancer patients receiving neoadjuvant chemotherapy by MR metabolic profiling. NMR Biomed 25: 369-378.

Chang JM, Moon WK, Cho N, Yi A, Ryoung Koo H, Han W, Noh D, Moon H, Kim S (2011) Clinical application of shear wave elastography (SWE) in the diagnosis of benign and malignant breast diseases. Breast Cancer Res Treat 129: 89-97.

Chen LC, Tu SH, Huang CS, Chen CS, Ho CT, Lin HW, Lee CH, Chang HW, Chang CH, Wu CH, Lee WS, Ho YS (2012) Human breast cancer cell metastasis is attenuated by lysyl oxidase inhibitors through down-regulation of focal adhesion kinase and the paxillin-signaling pathway. Breast Cancer Res Treat 134: 989-1004.

Cosgrove DO, Berg WA, Dore CJ, Skyba D, Henry J, Gay J, Cohen-Bacrie C (2012) Shear wave elastography for breast masses is highly reproducible. Eur Radiol 22: 1023-1032.

Desmedt C, Di Leo A, De Azambuja E, Larsimont D, Haibe-Kains B, Selleslags J, Delaloge S, Duhem C, Kains JP, Carly B, Maerevoet M, Vindevoghel A, Rouas G, Lallemand F, Durbecq V, Cardoso F, Salgado R, Rovere R, Bontempi G, Michiels S, Buyse M, Nogaret JM, Qi Y, Symmans F, Pusztai L, D’Hondt V, Piccart-Gebhart M, Sotiriou C (2011) Multifactorial approach to predicting resistance to anthracyclines. J Clin Oncol 29: 1578-1586.

Dongfeng H, Daqing M, Erhu J (2012) Dynamic breast magnetic resonance imaging: pretreatment prediction of tumor response to neoadjuvant chemotherapy. Clin Breast Cancer 12: 94-101.

Evans A, Whelehan P, Thomson K, McLean D, Brauer K, Purdie C, Jordan L, Baker L, Thompson A (2010) Quantitative shear wave ultrasound elastography: initial experience in solid breast masses. Breast Cancer Res 12: R104.

Farmer P, Bonnefoi H, Anderle P, Cameron D, Wirapati P, Becette V, Andre S, Piccart M, Campone M, Brain E, MacGrogan G, Petit T, Jassem J, Bibeau F, Blot E, Bogaerts J, Aguet M, Bergh J, Iggo R, Delorenzi M (2009) A stroma-related gene signature predicts resistance to neoadjuvant chemotherapy in breast cancer. Nat Med 15: 68-74.

Hattangadi J, Park C, Rembert J, Klifa C, Hwang J, Gibbs J, Hylton N (2008) Breast stromal enhancement on MRI is associated with response to neoadjuvant chemotherapy. Am J Roentgenol 190: 1630-1636.

Hatzis C, Pusztai L, Valero V, Booser DJ, Esserman L, Lluch A, Vidaurre T, Holmes F, Souchon E, Wang H, Martin M, Cotrina J, Gomez H, Hubbard R, Chacón JI, Ferrer-Lozano J, Dyer R, Buxton M, Gong Y, Wu Y, Ibrahim N, Andreopoulou E, Ueno NT, Hunt K, Yang W, Nazario A, DeMichele A, O'Shaughnessy J, Hortobagyi GN, Symmans WF (2011) A genomic predictor of response and survival following taxane-anthracycline chemotherapy for invasive breast cancer. JAMA 305: 1873-1881. 
Hayashi M, Yamamoto Y, Ibusuki M, Fujiwara S, Yamamoto S, Tomita S, Nakano M, Murakami K, Iyama KI, Iwase H (2012) Evaluation of tumor stiffness by elastography is predictive for pathologic complete response to neoadjuvant chemotherapy in patients with breast cancer. Ann Surg Oncol 19: 3042-3049.

Levental KR, Yu H, Kass L, Lakins JN, Egeblad M, Erler JT, Fong SF, Csiszar K, Giaccia A, Weninger W, Yamauchi M, Gasser DL, Weaver VM (2009) Matrix crosslinking forces tumor progression by enhancing integrin signaling. Cell 139: 891-906.

Lewis RA, Rogers KD, Hall CJ, Towns-Andrews E, Slawson S, Evans A, Pinder SE, Ellis IO, Boggis CR, Hufton AP, Dance DR (2000) Breast cancer diagnosis using scattered X-rays. J Synchrotron Radiat 7: 348-352.

Lips EH, Mulder L, De Ronde JJ, Mandjes IA, Vincent A, Vrancken Peeters MT, Nederlof PM, Wesseling J, Rodenhuis S (2012) Neoadjuvant chemotherapy in ER + HER2- breast cancer: response prediction based on immunohistochemical and molecular characteristics. Breast Cancer Res Treat 131: 827-836.

National Research Ethics Service (2008) Approval for Medical Devices Research: Guidance for Researchers, Manufacturers, Research Ethics Committees and NHS R\&D Offices (Version 2). National Patient Safety Agency: London.
Symmans WF, Peintinger F, Hatzis C, Rajan R, Kuerer H, Valero V, Assad L, Poniecka A, Hennessy B, Green M, Buzdar AU, Singletary SE, Hortobagyi GN, Pusztai L (2007) Measurement of residual breast cancer burden to predict survival after neoadjuvant chemotherapy. J Clin Oncol 25: 4414-4422.

Tokes AM, Szasz AM, Farkas A, Toth AI, Dank M, Harsanyi L, Molnar BA, Molnar IA, Laszlo Z, Rusz Z, Kulka J (2009) Stromal matrix protein expression following preoperative systemic therapy in breast cancer. Clin Cancer Res 15: 731-739.

Witkiewicz AK, Dasgupta A, Sotgia F, Mercier I, Pestell RG, Sabel M, Kleer CG, Brody JR, Lisanti MP (2009) An absence of stromal caveolin-1 expression predicts early tumor recurrence and poor clinical outcome in human breast cancers. Am J Pathol 174: 2023-2034.

This work is published under the standard license to publish agreement. After 12 months the work will become freely available and the license terms will switch to a Creative Commons AttributionNonCommercial-Share Alike 3.0 Unported License. 